\title{
A programmable energy efficient readout chip for a multiparameter highly integrated implantable biosensor system
}

\author{
M. Nawito ${ }^{1}$, H. Richter ${ }^{1}$, A. Stett ${ }^{2}$, and J. N. Burghartz ${ }^{1}$ \\ ${ }^{1}$ Institut für Mikroelektronik Stuttgart, Stuttgart, Germany \\ ${ }^{2}$ NMI Naturwissenschaftliches und Medizinisches Institut an der Universität Tübingen, Reutlingen, Germany \\ Correspondence to: M. Nawito (nawito@ims-chips.de)
}

Received: 15 December 2014 - Revised: 9 April 2015 - Accepted: 15 July 2015 - Published: 3 November 2015

\begin{abstract}
In this work an Application Specific Integrated Circuit (ASIC) for an implantable electrochemical biosensor system (SMART implant, Stett et al., 2014) is presented. The ASIC drives the measurement electrodes and performs amperometric measurements for determining the oxygen concentration, potentiometric measurements for evaluating the pH-level as well as temperature measurements. A 10-bit pipeline analog to digital (ADC) is used to digitize the acquired analog samples and is implemented as a single stage to reduce power consumption and chip area. For $\mathrm{pH}$ measurements, an offset subtraction technique is employed to raise the resolution to 12-bits. Charge integration is utilized for oxygen and temperature measurements with the capability to cover current ranges between $30 \mathrm{nA}$ and $1 \mu \mathrm{A}$. In order to achieve good performance over a wide range of supply and process variations, internal reference voltages are generated from a programmable band-gap regulated circuit and biasing currents are supplied from a wide-range bootstrap current reference. To accommodate the limited available electrical power, all components are designed for low power operation. Also a sequential operation approach is applied, in which essential circuit building blocks are time multiplexed between different measurement types. All measurement sequences and parameters are programmable and can be adjusted for different tissues and media. The chip communicates with external unites through a full duplex two-wire Serial Peripheral Interface (SPI), which receives operational instructions and at the same time outputs the internally stored measurement data. The circuit has been fabricated in a standard 0.5- $\mu \mathrm{m}$ CMOS process and operates on a supply as low as $2.7 \mathrm{~V}$. Measurement results show good performance and agree with circuit simulation. It consumes a maximum of $500 \mu \mathrm{A} \mathrm{DC}$ current and is clocked between $500 \mathrm{kHz}$ and
\end{abstract}

$4 \mathrm{MHz}$ according to the measurement parameters. Measurement results of the on-chip ADC show a Differential Non Linearity (DNL) lower than 0.5 LSB, an Integral Non Linearity (INL) lower than $1 \mathrm{LSB}$ and a Figure of Merit (FOM) of $6 \mathrm{pJ} /$ conversion.

\section{Introduction}

An integral part of all types of active implantable medical devices, such as cochlear and brain implants, is the electronic module. For monitoring of neuronal and metabolic activity a readout chip has to be implemented, which controls the data acquisition and management. In case of biosensor applications like subcutaneous metabolic monitoring, the electrochemical detection of ions, oxygen and $\mathrm{pH}$ requires a precise setting and measurement of voltage and currents at the metallic microelectrodes (Kubon et al., 2010; Jafari et al., 2014). For applications where large batteries and cabling is not suitable, stringent requirements on the readout chip in terms of size and energy efficiency are placed.

The SMART Implant consortium develops highly integrated implantable biosensor systems (Stett et al., 2014). As shown in Fig. 1a, the system contains a Read-Out Application Specific Integrated Circuit (RO-ASIC, or ROIC) which controls the measurement electrodes connected directly to the tissue or material to be characterized. A microcontroller sends instructions to the ROIC, receives the results and relays them to the power and data management ASIC. This frontend chip is responsible for power supply regulation of the implant and for the transmission of data via an inductive interface to an external reader unit placed outside the body. In this paper, the read out ASIC developed for this system is 


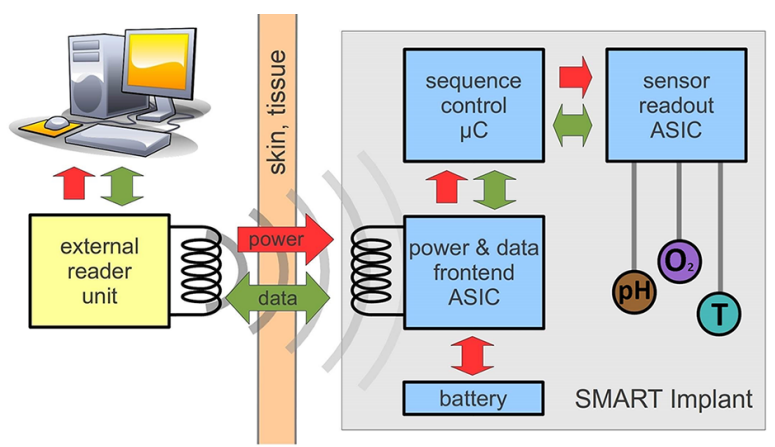

(a)

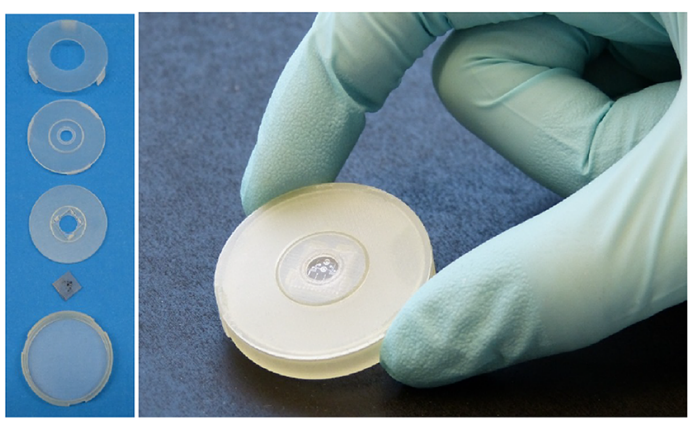

(b)

Figure 1. (a) Block diagram of the SMART Implant system highlighting readout ASIC, (b) photograph of actual implant and housing structure.

presented. The aim here is not to go through all the numerous analog and digital circuit blocks individually, but rather to give a description of the chip's functionality and structure, highlighting issues of energy efficiency, programmability and reliability of operation, in addition to the design techniques employed to approach these aspects.

\section{Measurement sequence}

The ROIC drives the measurement electrodes and performs amperometric measurements for determining the oxygen concentration, potentiometric measurements for evaluating the $\mathrm{pH}$-level as well as temperature measurements. In order to increase the data integrity of the measurement process, a sequential approach has been adopted, hence avoiding any disturbance that might occur due to simultaneous sampling and processing of different signals. To further improve the quality of the acquired data, a number of up to 128 "single measurements" are preformed and then averaged in order to obtain a measurement sample. This way random spikes or erratic data points are eliminated, which are generated due the fluctuating nature of the chemical reaction taking place between the electrode surface and the connected tissue (Lindner et al., 1986). The measurement samples form a "measurement sequence", which would eventually settle to a final value. The number of samples and the final value are determined by the external microcontroller according to the criteria set by the International Union of Pure and Applied Chemistry (IUPAC; Lindner et al., 1986). The aforementioned concept is illustrated in Fig. 2. As shown, it is a requirement for single measurements, whether they are of temperature, $\mathrm{pH}$ or $\mathrm{O}_{2}$ type, to be performed at least every $128 \mu$ s, but the time scale for a final value to be reached is in the order of seconds. The chip performs a complete measurement sequence each $15 \mathrm{~min}$. Figure 2 also emphasizes the necessity for an energy efficient design since the ROIC is supposed to remain operational inside the battery operated implant for duration up to 4 weeks.

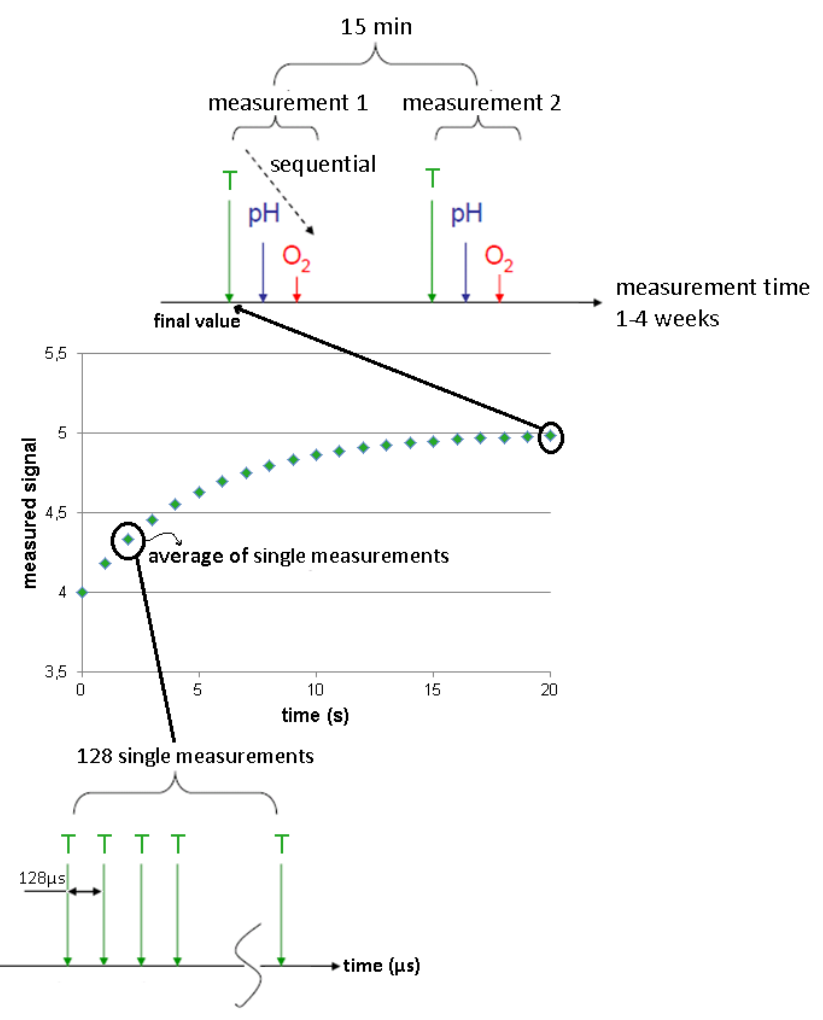

Figure 2. Measurement cycle as performed by the ROIC.

Since it is an essential requirement for the implant to be able to measure and characterize different types of tissues and materials, the ROIC must be designed to allow for a programmable and reliable operation. For this reason a parameterized approach has been adopted, where all measurement sequences and types can be carried out with different run times, boundary conditions and settings. Also an obstacle facing in vivo biosensors is the lack of access to the measured media, or to put it simply, the sensors are measuring in the dark, with no possibility to detect, observe or see the mea- 


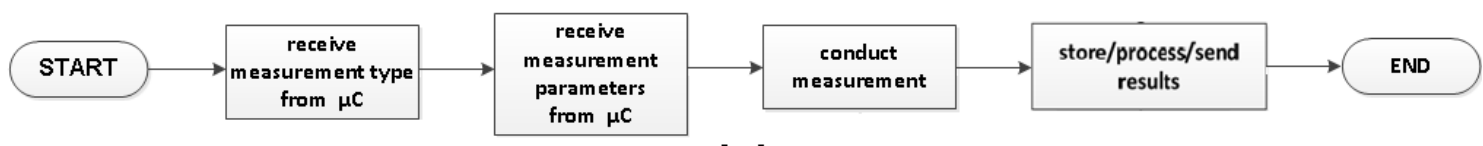

(a)

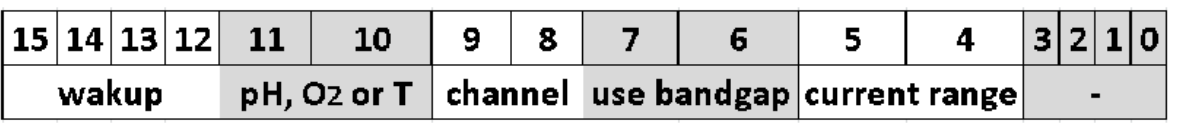

(b)

Figure 3. (a) Flow chart of the measurement sequence, (b) wakeup opcode and relevant parameters. Upon wakeup the measurement type is set, the input channels and current range are set and the bandgap circuit is either turned on or off.

surement conditions. For this reason the calibration and presetting of the ROIC is imperative to allow for meaningful interpretation of the results. For example when performing $\mathrm{O}_{2}$ measurements, the settling time required for the electrodes to start producing measurable currents differs from one material to another, hence the settling time is calibrated according to the tissue to be characterized.

Figure 3a shows the implemented flow chart of the ROIC. The chip receives a 16 bit operational code or opcode, where the first 4 bits constitute the instruction to be carried out (measure, calibrate, sleep etc.) and the relevant parameters are packed in the remaining 12 bits. As an example, Fig. 3b illustrates the structure of the wakeup command and the parameters passed to the chip during wakeup process. As seen during wakeup the measurement type and measurement channels are chosen, in addition to the setting of the current measurement range for $\mathrm{O}_{2}$ and temperature measurements. Also for testing and calibration purposes, reference voltages of the ADC could be either controlled by a bandgap circuit or generated directly from a simple resistive divider, hence the option "use bandgap" is available during wakeup.

\section{ASIC structure}

To implement the functions described in the previous section, the ASIC has been divided into digital and analog sections, as shown in Fig. 4. For both $\mathrm{O}_{2}$ and $\mathrm{pH}$ measurements, two measurement channels have been implemented respectively.

\subsection{Digital circuits}

For communication, a full duplex Serial Peripheral Interface (SPI) is implemented allowing the chip to simultaneously receive the 16 bit opcode from the external microcontroller and to send measurement results as a 16 bit output data word. The digital controller interprets the received opcodes and activates the analog circuitry which drives the external electrodes connected to the tissue and carry out the actual measurement. In case of a data transmission error or a faulty opcode, the chip sends an interrupt signal to request a resending of the instructions. To realize an energy efficient operation, all measurements are conducted with only the needed analog blocks turned on. For example since the charge integrator, as will be discussed shortly, is only required for amperometric sensing, it is turned off during potentiometric measurements. In the case of idle times where the chip is not measuring, all components are turned off except for the SPI interface which continues to listen for incoming instructions.

\subsection{ADC}

A central component of the readout path is a 10 bit cyclic ADC based on the pipelined principle, which converts the measured signal and sends it to the digital core for processing and storage. The ADC is designed to convert input voltages between 0.5 and $2.5 \mathrm{~V}$ with a LSB of $1.96 \mathrm{mV}$. The pipelined architecture has been chosen due to its relatively simple circuitry, reliable operation and capability of achieving the required 10 bit resolution needed for temperature and $\mathrm{O}_{2}$ measurements. A typical structure for such an ADC would consist of 9 stages in series, each producing 1.5 bits, and some form of digital correction. However, the large chip area occupied by such an arrangement in addition to its high power consumption would be unacceptable for this application, which necessitated a modified design. Figure 5 shows the implemented ADC which consists of a single 1.5 bit stage followed by a sample and hold circuit. The input analog signal is converted by the sub ADC, the residue is sampled by the sample and hold stage and then same circuit is reused again until the 10 bit word is produced. In other words instead of converting the analog input through 9 stages, a single stages is reused 9 times, reducing the area and power consumption by almost an order of magnitude.

\section{$3.3 \mathrm{pH}$ measurement}

For the $\mathrm{pH}$ measurements, a 12 bit resolution was required. To achieve this without redesigning the entire ADC and increasing the complexity of the design, an offset subtraction technique is introduced along with a couple of extra components to the 1.5 bit stage circuit. Specifically the voltage gen- 


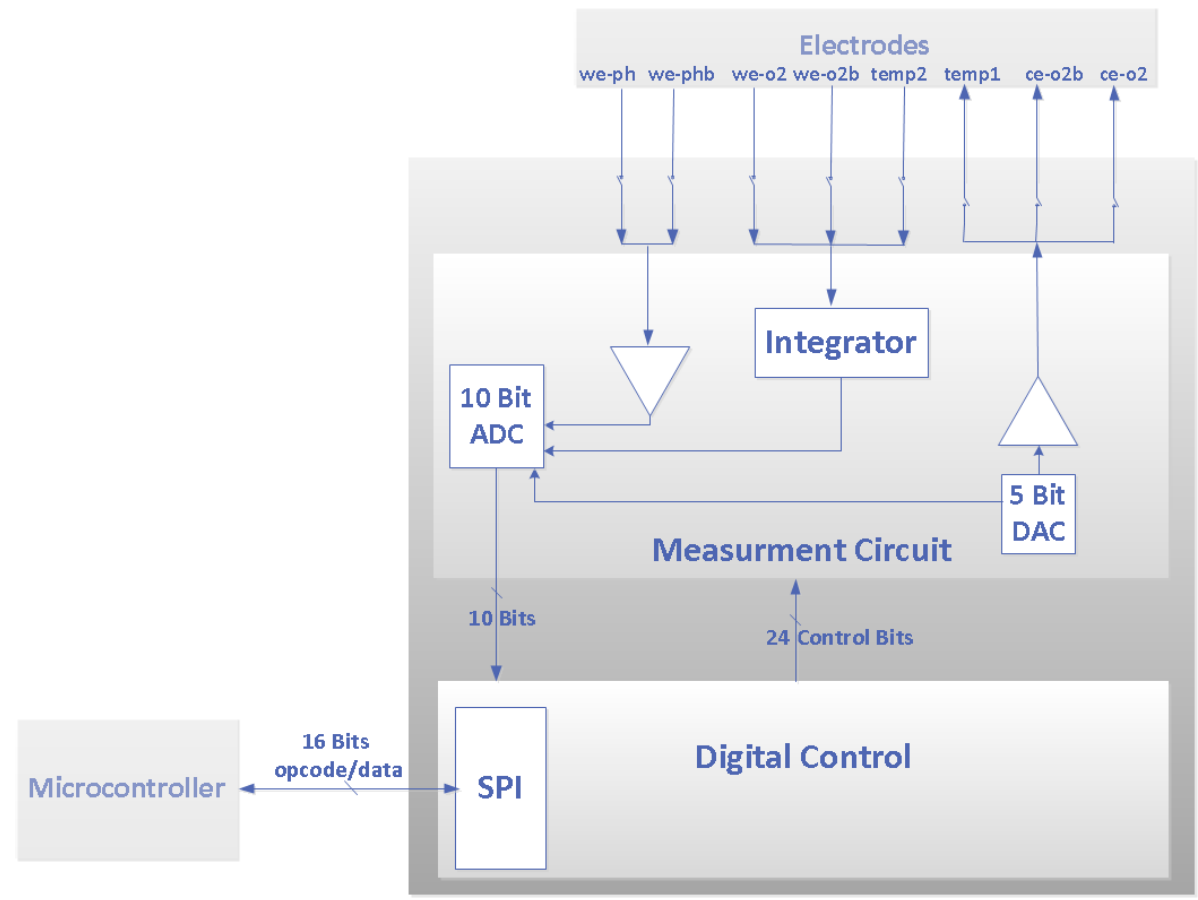

Figure 4. ROIC block diagram.

1.5 bit stage

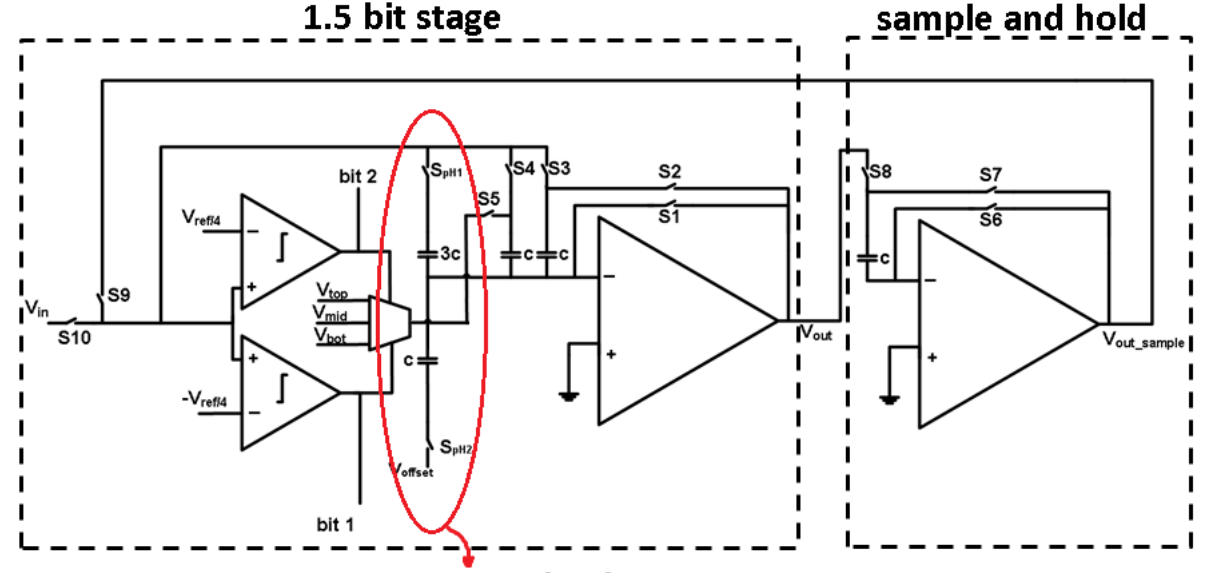

pH mode elements

Figure 5. ADC circuit with special pH mode switches and capacitors.

erated by the measurement electrodes, which corresponds to the measured $\mathrm{pH}$ value and lays between 0.7 and $2.3 \mathrm{~V}$, is first converted to a 10 bit "coarse" word, then according to the conversion result, a known offset generated by the on-chip 5-bit Digital to Analog Converter (DAC) (shown in Fig. 4) is subtracted from the original input. The difference is multiplied by 4 and converted to a 10 bit "fine" word. Summing the value of the offset and the fine results gives the final value with the required 12 bit resolution. To realize the aforementioned process, switches Sph1 and Sph2, in addition to capacitors with the values $C$ and $3 C$ are added as a new modi- fication to the circuit, as highlighted in Fig. 5, since they are specifically used to carry out the offset subtraction and difference multiplication. Specifically, in the first phase of the $\mathrm{pH}$ mode operation, switches are clocked so that the multiplying DAC connecting these elements with is connected as shown in Fig. 6a, where in that case the total charge of the system is given by

$Q_{\text {phase } 1}=V_{\text {in }} \times 3 C$. 


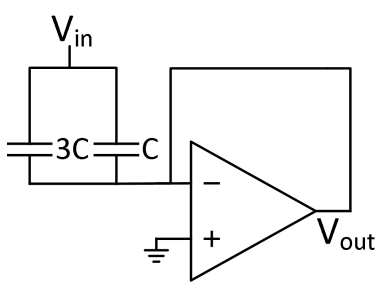

(a)

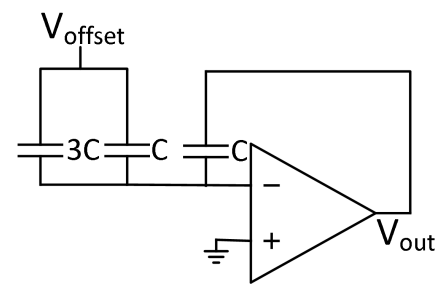

(b)
Figure 6. (a) $\mathrm{pH}$ mode phase 1, (b) $\mathrm{pH}$ mode phase 2 .

In phase 2, the circuit is configured as shown in Fig. $6 \mathrm{~b}$ and the total charge is given by

$Q_{\text {phase } 2}=V_{\text {out }} \times C+V_{\text {offset }} \times 4 C$.

Since $Q_{\text {phase1 }}$ equals $Q_{\text {phase2 }}$ the final output voltage is given by

$V_{\text {out }}=4\left(V_{\text {in }}-V_{\text {offset }}\right)$.

\section{$3.4 \mathrm{O}_{2}$ and temperature measurement}

In the case of $\mathrm{O}_{2}$ and temperature measurement, the measured analog signals are currents, where for the former a three electrode measurement setup is implemented and current flowing between the working electrode and the counter electrode is of interest (Kubon et al., 2010). For temperature measurements, an external Schottky diode is reversed biased and used as a transducer, where the reverse current is a measure of the temperature and the sensitivity of the sensor is controlled by the reverse bias voltage. The reverse voltage is also produced by the internal DAC and is one of the parameters to be set by the user.

To convert currents into voltages, a necessary step given that the ADC operates on voltage inputs, the charge integrator shown in Fig. 6. is implemented. Following the basic equation of charge integration which states that

$V_{\text {out }}=\frac{I_{\text {in }} \times t}{C}$,

where $V_{\text {out }}$ is the output voltage, $I_{\text {in }}$ is the input current, $t$ is the integration time and $C$ is the integration capacitance, it is clear that by adjusting $t$ and $C$, various current ranges can be measured. For this reason, different integration capacitances are added in parallel, as illustrated in Fig. 7. Furthermore, the integration time can be set as an input parameter, allowing the implemented integrator to cover current ranges between $30 \mathrm{nA}$ and $1 \mu \mathrm{A}$ with a 10 bit resolution.

\section{Fabrication}

The design has been implemented using IMS GATE FOREST $^{\circledR} \quad 0.5 \mu \mathrm{m}, 2$ Metal CMOS technology

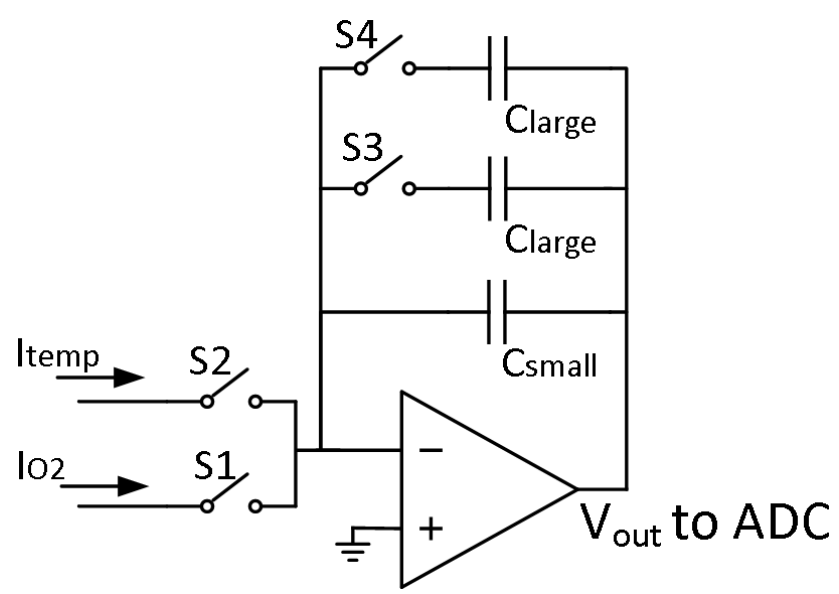

Figure 7. Programmable charge integrator.

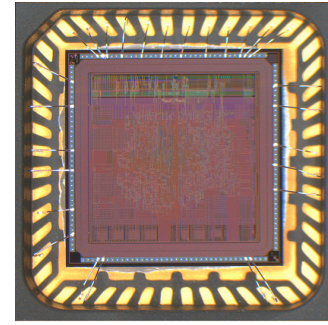

(a)

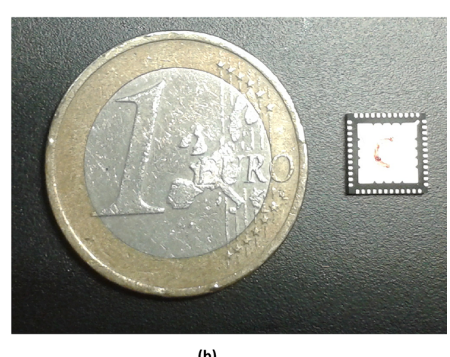

(b)
Figure 8. (a) Photograph of fabricated chip in housing, (b) size comparison between final chip and a coin.

(http://www.ims-chips.de/content/pdftext/White_paper_ MS_Array_09_11.pdf). This sea of transistors technology offers transistors and passive circuit elements implemented in a certain number of fixed dimensions. The digital and ana$\log$ circuits are then realized by connecting these elements with metal wires creating semi custom designs. A major advantage of this approach is that the devices are very well characterized and modeled, enhancing the process yield and the reliability of the ASIC.

In Fig. 8a, a photograph of the fabricated ASIC bonded inside a QFN48 housing is shown, where Fig. 8b shows a size comparison between the final sealed housing to be used in the actual implant and a coin.

\section{Measurement results}

In Fig. 9a and b, the measured Differential Nonlinearity (DNL) and measured Integral Nonlinearity (INL) of the on chip ADC are shown, respectively. The test setup for producing the measured results involved connecting a voltage sources to the working electrodes of the $\mathrm{pH}$ circuits, sweeping the voltage and evaluating the digital output data sent via the SPI interface. The ADC also demonstrated a Figure of Merit (FOM) of $6 \mathrm{pJ} /$ conversion. 


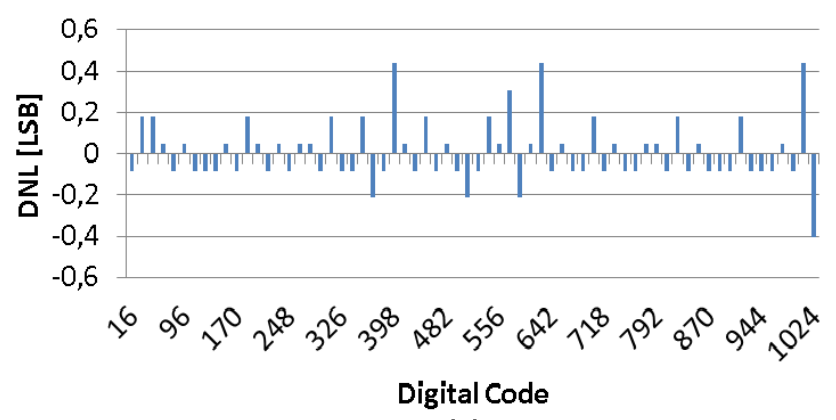

(a)

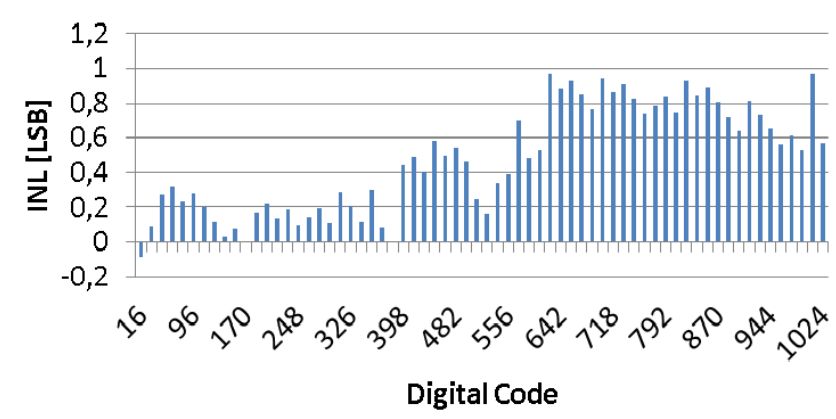

(b)

Figure 9. (a) Measured ADC DNL, (b) measured ADC INL.

Table 1. Performance parameters of the ROIC.

\begin{tabular}{ll}
\hline Technology & $\begin{array}{l}0.5 \mu \mathrm{m}, 2 \mathrm{M} \text { CMOS, } \\
\text { see of gates } \\
\end{array}$ \\
$\begin{array}{l}\text { Supply } \\
\text { Temperature }\end{array}$ & $20-3.3 \mathrm{~V}$ \\
Max. total DC current & $\sim 500 \mu \mathrm{C}$ at $3.3 \mathrm{~V}, 80^{\circ} \mathrm{C}$ \\
Min. total DC current & $\sim 250 \mu \mathrm{A}$ at $2.7 \mathrm{~V}, 20^{\circ} \mathrm{C}$ \\
Nominal operating frequency & $500 \mathrm{kHz}$ \\
Max. operating frequency & $4 \mathrm{MHz}$ \\
Number of analog devices & 432 \\
Number of digital gates & 1185 \\
(NAND equiv.) & \\
\hline
\end{tabular}

The chip operates at supply voltages between 2.7 and $3.3 \mathrm{~V}$ and at temperatures between 20 and $80^{\circ} \mathrm{C}$, where the temperature measurement circuits have been optimized for the range between 20 and $43^{\circ} \mathrm{C}$ with an accuracy of $0.1^{\circ} \mathrm{C}$. The maximum total DC current that all circuits could consume is about $500 \mu \mathrm{A}$ nevertheless, this value is never reached since that would require all digital and analog building blocks to be turned on at the same time. As mentioned before, this case is avoided by activating the relevant components for a certain measurement type only. In order to reduce dynamic power consumption and at the same time fulfilling the requirement of obtaining a single measurement point every $128 \mu$ s given the cyclic nature of the ADC, a nominal operating frequency of $500 \mathrm{kHz}$ is chosen. However, the chip can function reliably at a maximum frequency of up to
$4 \mathrm{MHz}$. Table 1 summarizes the performance parameters of the ROIC.

\section{Conclusion}

The successful design and implementation of a programmable read out ASIC for miniature active implants comprising high functionality is an interesting and multifaceted undertaking. The core philosophy driving the development process should be the consideration of energy requirements and the fulfilment of flexible operation during all phases of the design. Efficient scheduling of the measurement sequence, implementing an elaborate set of operations and the time multiplexing of internal components, in addition to the optimization of power consumption on the circuit level are all important methods in their own right, but yield the most interesting results when employed in combination.

Acknowledgements. This work was funded by the German Federal Ministry of Education and Research, BMBF as part of the MicroTEC Südwest Cluster project SMART Implant, grant no. 16SV5979K, 16SV5980 and 16SV5982-86. The authors would also like to sincerely thank all members and partners of the SMARTImplant consortium. Without their valuable efforts, this work would not have been possible.

Edited by: D. Killat

Reviewed by: two anonymous referees

\section{References}

Jafari, H. M., Abdelhalim, K., Soleymani, L., Sargent, E. H., Kelley, S. O., and Genov, R.: Nanostructured CMOS Wireless UltraWideband Label-Free PCR-Free DNA Analysis SoC, IEEE Journal of Solid-State Circuits, 49, 1223-1241, 2014.

Kubon, M., Moschallski, M., Link, G., Ensslen, T., Werner, S., Burkhardt, C., Nisch, W., Scholz, B., Schlosshauer, B., Urban, G., and Stelzle, M.: A Microsensor System to Probe Physiological Environments and Tissue Response, IEEE Conference on Sensors, Kona, Hawaii, 1-4 November 2010, 2607-2611, 2010.

Lindner, E., Toth, K., and Pungor, E.: Definition and determination of response time of ion selective electrodes, Pure Appl. Chem, 58, 469-479, 1986.

Stett, A., Bucher, V., Cihova, M., Gutoehrlein, K., Kubon, M., Link, G., von Metzen, R., Stamm, B., Stelzle, M., Pojtinger, A., Schneider, K., Mintenbeck, D., Rossbach, D., Richter, H., Nawito, M., Boven, K.-H.,; Moeller, A., Jeschke, C., Paetzold, J., Goettsche, T., Bludau, O., Haas, N., Tompkins, D., Lebold, T., and Kokelmann, M.: SMART Implant: Electronic Implants for Diagnosis and Monitoring, Energieautarke Sensorik (GMM-FB 79), Contributions of the 7th GMM Workshop, Magdeburg, Germany, 24-25 February, 2014. 